\title{
Enteric methane emissions, energy partitioning, and energetic efficiency of zebu beef cattle fed total mixed ration silage
}

\author{
Sayan Subepang ${ }^{1}$, Tomoyuki Suzuki ${ }^{2}$, Thamrongsak Phonbumrung ${ }^{3}$, and Kritapon Sommart ${ }^{1, *}$
}

* Corresponding Author: Kritapon Sommart Tel: +66-432-02360, Fax: +66-432-02361,

E-mail: kritapon@kku.ac.th

' Department of Animal Science, Faculty of Agriculture, Khon Kaen University, Khon Kaen 40002, Thailand

2 Japan International Research Center for Agricultural

Science, Tsukuba, Ibaraki 305-8586, Japan

${ }^{3}$ Bureau of Animal Nutrition Development,

Department of Livestock Development, Ratchathewi, Bangkok 10400, Thailand

ORCID

Sayan Subepang

https://orcid.org/0000-0002-3667-6606

Tomoyuki Suzuki

https://orcid.org/0000-0002-5313-7647

Thamrongsak Phonbumrung

https://orcid.org/0000-0001-7853-5417

Kritapon Sommart

https://orcid.org/0000-0002-6947-412X

Submitted Jun 5, 2018; Revised Jul 23, 2018; Accepted Sept 3, 2018
Objective: The main objective of this study was to evaluate the effect of different feeding levels of a total mixed ration silage-based diet on feed intake, total tract digestion, enteric methane emissions, and energy partitioning in two beef cattle genotypes.

Methods: Six mature bulls (three Thai natives, and three Thai natives - Charolais crossbreeds) were assigned in a replicated $3 \times 3$ Latin square design, with cattle breed genotype in separate squares, three periods of 21 days, and three energy feeding above maintenance levels $(1.1,1.5$, and 2.0 MEm, where MEm is metabolizable energy requirement for maintenance). Bulls were placed in a metabolic cage equipped with a ventilated head box respiration system to evaluate digestibility, record respiration gases, and determine energy balance.

Results: Increasing the feeding level had no significant effect on digestibility but drastically reduced the enteric methane emission rate $(\mathrm{p}<0.05)$. Increasing the feeding level also significantly increased the energy retention and utilization efficiency $(\mathrm{p}<0.01)$. The Thai native cattle had greater enteric methane emission rate, digestibility, and energy utilization efficiency than the Charolais crossbred cattle $(\mathrm{p}<0.05)$. The daily metabolizable energy requirement for maintenance in Thai native cattle $\left(388 \mathrm{~kJ} / \mathrm{kg} \mathrm{BW}{ }^{0.75}\right.$, where $\mathrm{BW}^{0.75}$ is metabolic body weight) was $15 \%$ less than that in Charolais crossbred cattle $\left(444 \mathrm{~kJ} / \mathrm{kg} \mathrm{BW}^{0.75}\right)$.

Conclusion: Our results suggested that the greater feeding level in zebu beef cattle fed above maintenance levels resulted in improved energy retention and utilization efficiency because of the reduction in enteric methane energy loss. The results also indicated higher efficiency of metabolisable energy utilization for growth and a lower energy requirement for maintenance in Bos indicus than in Bos taurus.

Keywords: Digestibility; Energy Balance; Greenhouse Gas; Zebu; Ruminants

\section{INTRODUCTION}

Energy loss in ruminant livestock through enteric methane emissions is a problem not only because of the impact on climate change but also owing to the considerable effect on animal productivity. Ruminant animals, particularly cattle (Bos taurus [B. taurus] and Bos indicus [B. indicus] ), produce significant amounts of methane via anaerobic gut digestion. Compared to other ruminants, beef and dairy cattle contribute the most to methane emissions due to their greater body size, energy intake, and population size; they produce $61 \%$ of the emissions attributed to all domestic animals [1] and cause a loss of enteric methane energy accounting for $2 \%$ to $12 \%$ of gross energy (GE) intake. In addition, methane energy loss reduces the efficiency of feed energy utilization and beef cattle productivity [2]. Therefore, the use of feeding strategies to reduce enteric methane emissions is a priority in improving animal productivity and environmental sustainability [3].

Zebu cattle (B. indicus; also referred to in this report as Thai native cattle) and their crossbreeds with European cattle (B. taurus) provide the main genotypes for beef cattle populations 
because they are considered to be well adapted to heat stress, disease, and the low quality feed found in conditions of humid tropical environments. Crossbreeding has been widely adopted to improve growth performance and meat quality, given the advantages for commercialization in the beef industry of genetic heterosis and the complementarity between high productivity and hot climates. We hypothesized that $B$. indicus $\times B$. taurus crossbred beef cattle have a higher energy requirement than $B$. indicus purebred zebu beef cattle. This is important because the energy requirement is a function of energetic efficiency that determines the energy supply required to meet production targets [4-6]. Our previous studies have reported that a lower energy is required for maintaining zebu than for European beef cattle [2,7]. However, little research has been undertaken on the energetic efficiency and enteric methane emissions in $B$. indicus $\times B$. taurus crossbred beef cattle under tropical humid conditions. In particular, there are no data available comparing the energy balance between Thai native cattle ( $B$. indicus) and Charolais crossbred cattle (B. indicus $\times$ B. taurus). Therefore, the objective of this research was to determine the effect of feeding levels on feed intake, digestibility, enteric methane emissions, energy partitioning, and the efficiency of metabolizable energy (ME) utilization in zebu beef cattle fed total mixed ration silage.

\section{MATERIALS AND METHODS}

\section{Experiment location and animal care}

The experiment was conducted at Khon Kaen University Farm Research Station, Khon Kaen province, Thailand $\left(16.46^{\circ} \mathrm{N}\right.$ $102.82^{\circ} \mathrm{E}$; altitude $169 \mathrm{~m}$ above sea level). The management of cattle used in the study and all related procedures were performed according to the Guidelines of the Ethics of Animal Experimentation of the National Research Council of Thailand, with permission of the Animal Ethics Committee of Khon Kaen University (Record No. AEKKU23/2557, Reference No. 0514.1.12.2/27).

\section{Animals, diet, and experimental design}

Animals used in the study were three 2.5 year old Thai native bulls and three 2.0 year old Charolais crossbred bulls (50\% Charolais $\times 25 \%$ Brahman $\times 25 \%$ Thai native cattle) with average body weights of $310 \pm 12.8 \mathrm{~kg}$ (mean \pm standard deviation) and $369 \pm 32.1 \mathrm{~kg}$ (mean \pm standard deviation), respectively. The experiment employed a replicated $3 \times 3$ Latin square design, assigning the cattle breed genotype in a separate square with three periods (21 days per period) and three dietary treatment feeding levels (1.1, 1.5, and 2.0 MEm; MEm represents the ME requirement for maintaining beef cattle that is equal to 486 $\mathrm{kJ} / \mathrm{kg}$ body weight $(\mathrm{BW})^{0.75}$, where $\mathrm{BW}^{0.75}$ is metabolic body weight according to The Working Committee of Thai Feeding Standards for Ruminants (WTSR) [6]). The animals were housed individually in pens $(2.5 \times 4.5 \mathrm{~m})$, fed at 09:00 and 17:00 each day, and provided with clean drinking water.

The experimental diet was formulated to meet the nutrient requirements for beef cattle [6]: its analyzed chemical composition and feed ingredients are shown in Table 1. The diets were supplied in the form of an ensiled or fermented total mixed ration (FTMR). The FTMR was prepared by mixing a formulated ingredient ratio of $400 \mathrm{~kg}$ fresh matter per batch in a horizontal feed mixer (Pak Thong Chai Pasusat, Nakhon Ratchasima province, Thailand), and loading each batch into polyethylene silo bags $(1.50 \times 2.20 \mathrm{~m}, 0.14 \mathrm{~mm}$ thick; Sahavanit Industry Co., Ltd., Bangkok, Thailand). The silo bags were tightly packed using a commercial vacuum cleaner/blower (model 1800W VC-910; Imarflex Industrial Co., Ltd., Bangkok, Thailand), and after preparation were stored outdoors at approximately $25^{\circ} \mathrm{C}$ to $35^{\circ} \mathrm{C}$ for at least 15 ensiling days.

Table 1. Ingredients, analyzed chemical composition, fermentation profile, and energy content of the experimental fermented total mixed ration diet

\begin{tabular}{|c|c|}
\hline Items & Diet \\
\hline \multicolumn{2}{|l|}{ Ingredients (\% DM) } \\
\hline Rice straw & 20.0 \\
\hline Cassava pulp & 30.0 \\
\hline Coconut meal & 14.8 \\
\hline Palm kernel meal & 25.2 \\
\hline Rice bran & 8.5 \\
\hline Urea & 0.5 \\
\hline Minerals, mixed ${ }^{1)}$ & 0.5 \\
\hline Vitamins, premixed ${ }^{2)}$ & 0.5 \\
\hline Total $(\%)$ & 100.0 \\
\hline \multicolumn{2}{|l|}{ Chemical composition (\% DM) } \\
\hline Dry matter & 43.1 \\
\hline Organic matter & 93.5 \\
\hline Crude protein & 11.5 \\
\hline Ether extracts & 5.6 \\
\hline Neutral detergent fiber & 51.9 \\
\hline Acid detergent fiber & 32.5 \\
\hline \multicolumn{2}{|l|}{ Fermentation profile } \\
\hline $\mathrm{pH}$ & 3.7 \\
\hline Ammonia nitrogen ( $\%$ of DM) & 2.7 \\
\hline Lactic acid (\% of DM) & 4.1 \\
\hline Acetic acid (\% of DM) & 1.2 \\
\hline Propionic acid (\% of DM) & 0.04 \\
\hline Butyric acid (\% of DM) & 0.02 \\
\hline \multicolumn{2}{|l|}{ Energy content (MJ/kg DM) } \\
\hline Gross energy & 17.2 \\
\hline Digestible energy & 11.9 \\
\hline Metabolizable energy & 10.1 \\
\hline \multicolumn{2}{|c|}{$\begin{array}{l}\text { DM, dry matter; MJ, megajoules. } \\
\text { 1) Trace minerals premix provided the following per kg concentrate: cobalt, } 0.02 \mathrm{~g} \text {; } \\
\text { copper, } 1.60 \mathrm{~g} \text {; iodine, } 10.00 \mathrm{~g} \text {; manganese, } 8.00 \mathrm{~g} \text {; selenium, } 0.06 \mathrm{~g} ; \mathrm{zinc}, 6.00 \mathrm{~g} \text {; } \\
\text { anti-rancidity, } 2.50 \mathrm{~g} \text {; carrier, } 1,000.00 \mathrm{~g} \text {. } \\
\text { 2) Vitamins premix provided the following per kg concentrate: vitamin } \mathrm{A}, 2,000,000 \\
\mathrm{IU} \text {; vitamin } \mathrm{D}_{3}, 4,000,000 \mathrm{IU} \text {; vitamin } \mathrm{E}, 3,000 \mathrm{IU} \text {. }\end{array}$} \\
\hline
\end{tabular}




\section{Data and sample collection}

Cattle were weighed and recorded on the first and last day of each experimental period in the morning (07:30), to determine body weight and metabolic body weight for each feeding level.

Animals were moved to a metabolic cage for measurements of feed intake, digestibility, and respiratory gases. These measurements, which were made according to the method of Schneider and Flatt [8], were completed within six days of each collection period. Samples of both offered and refused feed, feces (1 kg), and urine (500 mL) containing $6 \mathrm{~N}$ (Normal) hydrochloric acid solution (to maintain a urine $\mathrm{pH}$ of $<3$ ) were sampled and weighed each morning for six days, and stored at $-18^{\circ} \mathrm{C}$ until analysis.

Respiratory gas exchange measurements were conducted during the last three days of the metabolic collection. Oxygen consumption and carbon dioxide and methane emissions for each animal were determined according to the method of Suzuki et al [9]. An indirect respiration calorimetry system, consisting of a ventilated head box (width $105 \mathrm{~cm} \times$ depth 80 $\mathrm{cm} \times$ height $173 \mathrm{~cm}$ ) and flow meter with a thermal flow cell (NFHY-R-O-U, Nippon Flow Cell Co., Ltd., Tokyo, Japan), was used to measure and record the flow rate and total air volume. A dual-chamber paramagnetic oxygen analyzer (Servopro 4100 Gas Purity Analyzer, Servomex Group, East Sussex, UK) was used to determine oxygen concentrations in the in- and outflow lines. An infrared gas analyzer (IR200 Infrared Gas Analyzer, Yokogawa Electric Corporation, Tokyo, Japan) was used to measure carbon dioxide and methane concentrations. The gas analyzers were calibrated daily with standard gases (Takachiho Chemical Industrial Co., Ltd., Tokyo, Japan). Calorimetric system recovery tests were conducted using the carbon dioxide injection method, by which a weighed amount of carbon dioxide gas was released into the system.

Energy partitioning, based on energy intake and energy loss through feces, urine, enteric methane, and heat production (HP) was determined according to the protocol of the Agricultural Research Council (ARC) [4]. The average of the antilog of the intercept of the linear regression between the log of $\mathrm{HP}$ and ME intake was used to estimate the efficiency of ME utilization for maintenance $\left(\mathrm{k}_{\mathrm{m}}\right)$. Energy retained (ER) was calculated by subtracting the HP from ME intake, and the linear regression of ER on ME intake produced the slope assumed to be the efficiency of energy utilization for growth $\left(\mathrm{k}_{\mathrm{g}}\right)$ and estimate MEm using ARC [10].

The fermentation profile and $\mathrm{pH}$ of the diet was determined using the technique described by Cao et al [11]. Ammonia nitrogen was measured [12] using a spectrometer (T80+ UV/ VIS Spectrometer, PG Instruments, London, UK), and volatile fatty acid and lactic acid content in the FTMR samples were determined using gas chromatography (GC-2014, Shimadzu, Kyoto, Japan), according to the method of Porter and Murray [13]. Samples of offered feed, refused feed, and feces were oven- dried at $65^{\circ} \mathrm{C}$ for $72 \mathrm{~h}$ and then ground in order to be passed through a $1 \mathrm{~mm}$ screen. AOAC procedures [14] were used to analyze dry feed and feces samples for dry matter (DM), ash, ether extracts, and crude protein (CP), (methods 967.03, 942.05, 920.39, and 984.13, respectively). Neutral detergent fiber (NDF), assayed with heat-stable amylase and expressed inclusively of residual ash and acid detergent fiber (ADF), was analyzed using the method of Van Soest et al [15]. Urine compounds were sampled to determine $\mathrm{N}$ content using the Kjeldahl procedure [14]. The GE of the feed, feces, and urine was determined using a bomb calorimeter (IKA Calorimeter System, C 2000 basic, IKA-Werke, Staufen, Germany).

\section{Statistical analysis}

All data were analyzed using the general linear model procedure of SAS [16] according to a replicated $3 \times 3$ Latin square design as follows: $Y_{\mathrm{ijkl}}=\mu+S_{1}+A_{i(l)}+\rho_{j}+\tau_{k}+\varepsilon_{i j k l}$, where $Y_{i j k l}$ is the mean response of cattle breed genotype 1 , cattle $i$, period $j$, treatment $\mathrm{k} ; \mathrm{S}_{\mathrm{l}}$, the effect of cattle breed genotype $(1=1$ to 2$) ; A_{\mathrm{i}(\mathrm{l})}$, the effect of cattle within cattle breed genotype ( $i=1$ to 6$) ; \rho_{j}$, the effect of the period ( $j=1$ to 3 ); $\tau_{k}$, the effect of treatment $(\mathrm{k}=1$ to 3$)$; and $\varepsilon_{\mathrm{ijkl}}$ is the random residual error. Because the interaction was not statistically significant ( $p>0.05)$, it was removed from the model. Linear and quadratic of contrast of the treatment means were estimated [17].

\section{RESULTS}

\section{Feed intake and digestibility}

Feed intake, digestibility, and growth performance are shown in Table 2. Charolais crossbred cattle had a higher daily intake of feed and nutrients ( $\mathrm{kg} \mathrm{DM}$ and $\left.\mathrm{g} / \mathrm{kg} \mathrm{BW}^{0.75}\right)$ than did Thai native cattle $(\mathrm{p}<0.01)$. Increasing the feeding level resulted in an increased daily intake of feed and nutrients $(\mathrm{p}<0.01)$. Thai native cattle showed higher values for DM and nutrient digestibility than Charolais crossbred cattle $(\mathrm{p}<0.05)$, and increasing the feeding level had no significant effect on digestibility of nutrients. Additionally, increasing feeding levels resulted in increases in body weight gain and average daily gain (ADG) $(\mathrm{p}<0.01)$, whereas Charolais crossbreeds had a higher growth performance than Thai native cattle $(\mathrm{p}<0.05)$.

\section{Enteric methane emissions}

Methane emission data are presented in Table 3. Charolais crossbred cattle emitted greater total enteric methane $(\mathrm{L} / \mathrm{d}$ and $\mathrm{MJ} / \mathrm{d}$ ) than Thai native cattle but, after correcting for metabolic body weight, no difference was observed ( $\mathrm{p}<0.05$; Table 4). The enteric methane emission rate (L/kg DM intake, $\mathrm{L} / \mathrm{kg}$ organic matter [OM] intake, and L/kg NDF intake; MJ/100 MJ GE intake) in Charolais crossbreeds was significantly less than that in Thai native cattle. Moreover, increasing the feeding level resulted in a significant linear reduction in the rate of 
Table 2. Daily feed intake, digestibility, and body weight in cattle fed a fermented total mixed ration at different feeding levels

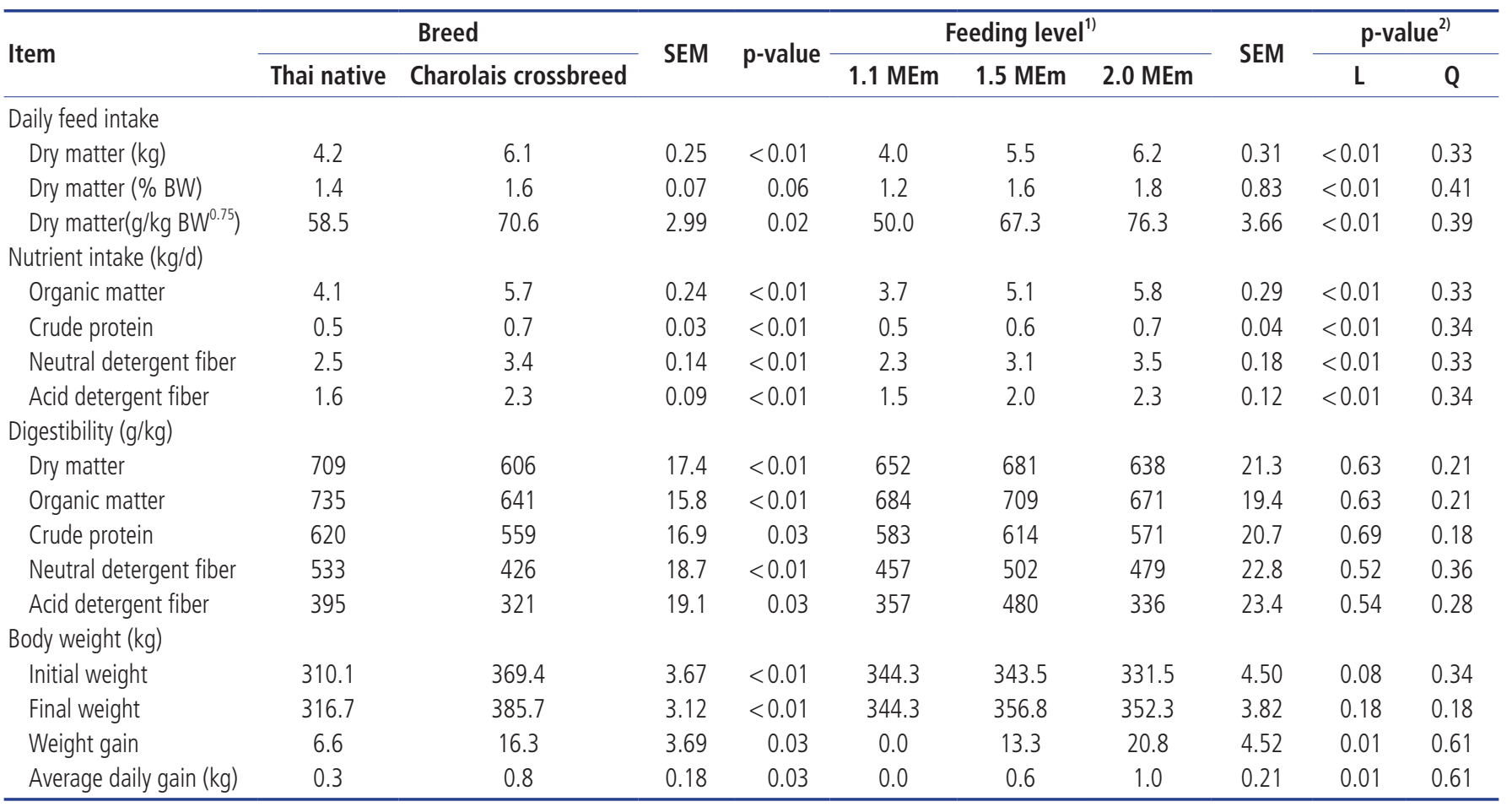

SEM, standard error of mean; BW, body weight; $\mathrm{BW}^{0.75}$; metabolic body weight.

${ }^{1)} \mathrm{MEm}$, metabolizable energy requirement for maintenance (486 kJ/kg BW0.75/d).

2) Polynomial contrast probability of a significant linear $(\mathrm{L})$ and quadratic $(\mathrm{Q})$ effect in the feeding levels.

enteric methane emissions, from $6.4 \%$ to $5.6 \%$ (MJ/100 MJ GE intake).

\section{Energy partitioning}

The energy partitioning results, expressed on the basis of metabolic body size and energy utilization of the cattle, are shown in Table 4. Compared to Thai native cattle, Charolais crossbreeds had a higher GE intake and energy loss in feces, urine, and HP. When corrected for metabolic body weight, digestible energy (DE) intake, ME intake, enteric methane production, and energy retention did not differ significantly between Thai native and Charolais crossbred cattle ( $\mathrm{p}>0.05)$.

Energy (GE, DE, and ME) intake and energy retention increased linearly $(\mathrm{p}<0.01)$ as feeding level increased. The proportions of ME to GE and ME to DE in Thai native cattle were higher than in Charolais crossbred cattle. Results for energy utilization $(\mathrm{MJ} / \mathrm{MJ})$ showed a linear increase $(\mathrm{p}<0.01)$ with increasing ME intake levels.

\section{Efficiency of metabolizable energy utilization}

The result for the regression of ME intake on energy retention was highly significant (Figure 1). The efficiency of $\mathrm{k}_{\mathrm{g}}$ was 0.65

Table 3. Enteric methane emissions from cattle fed a fermented total mixed ration at different feeding levels

\begin{tabular}{|c|c|c|c|c|c|c|c|c|c|c|}
\hline \multirow{2}{*}{ Item } & \multicolumn{2}{|r|}{ Breed } & \multirow{2}{*}{ SEM } & \multirow{2}{*}{ p-value } & \multicolumn{3}{|c|}{ Feeding level $^{1)}$} & \multirow{2}{*}{ SEM } & \multicolumn{2}{|c|}{$p$-value $e^{2)}$} \\
\hline & Thai native & Charolais crossbreed & & & 1.1 MEm & 1.5 MEm & 2.0 MEm & & L & Q \\
\hline \multicolumn{11}{|l|}{ Emission rate } \\
\hline $\mathrm{L} / \mathrm{d}$ & 118.9 & 149.0 & 6.03 & $<0.01$ & 109.7 & 142.1 & 150.1 & 7.38 & $<0.01$ & 0.22 \\
\hline L/kg DMI & 27.6 & 24.7 & 0.66 & 0.02 & 27.8 & 26.3 & 24.4 & 0.81 & 0.02 & 0.85 \\
\hline L/kg OMl & 29.5 & 26.4 & 0.71 & 0.02 & 29.7 & 28.1 & 26.1 & 0.87 & 0.02 & 0.85 \\
\hline $\mathrm{L} / \mathrm{kg} \mathrm{NDFl}$ & 48.7 & 43.6 & 1.12 & 0.02 & 49.0 & 46.4 & 43.0 & 1.43 & 0.02 & 0.85 \\
\hline L/kg ADG & 321.5 & 263.5 & 30.59 & 0.43 & 638.8 & 228.1 & 162.0 & 17.66 & 0.02 & 0.10 \\
\hline $\mathrm{MJ} / \mathrm{d}$ & 4.7 & 5.9 & 0.24 & $<0.01$ & 4.3 & 5.6 & 5.9 & 0.29 & $<0.01$ & 0.21 \\
\hline MJ/100 MJ GEl & 6.3 & 5.7 & 0.15 & 0.02 & 6.4 & 6.0 & 5.6 & 0.18 & 0.02 & 0.85 \\
\hline
\end{tabular}

SEM, standard error of mean; DMI, dry matter intake; OMI, organic matter intake; NDFI, neutral detergent fiber intake; ADG, average daily gain; GEl, gross energy intake.

${ }^{1)} \mathrm{MEm}$, metabolizable energy requirement for maintenance (486 $\left.\mathrm{kJ} / \mathrm{kg} \mathrm{BW} W^{0.75} / \mathrm{d}\right)$.

2) Polynomial contrast probability of a significant linear $(\mathrm{L})$ and quadratic $(\mathrm{Q})$ effect in the feeding levels. 
Table 4. Energy partitioning and utilization in cattle fed a fermented total mixed ration at different feeding levels

\begin{tabular}{|c|c|c|c|c|c|c|c|c|c|c|}
\hline \multirow{2}{*}{ Item } & \multicolumn{2}{|r|}{ Breed } & \multirow{2}{*}{ SEM } & \multirow{2}{*}{$p$-value } & \multicolumn{3}{|c|}{ Feeding levels ${ }^{1)}$} & \multirow{2}{*}{ SEM } & \multicolumn{2}{|c|}{$p$-value ${ }^{2)}$} \\
\hline & Thai native & Charolais crossbreed & & & 1.1 MEm & 1.5 MEm & 2.0 MEm & & $\mathbf{L}$ & Q \\
\hline \multicolumn{11}{|c|}{ Energy partition $\left(\mathrm{kJ} / \mathrm{kgBW}^{0.75} / \mathrm{d}\right)$} \\
\hline GE intake & $1,008.7$ & $1,221.3$ & 49.82 & 0.02 & 869.8 & $1,159.8$ & $1,315.4$ & 61.02 & $<0.01$ & 0.40 \\
\hline DE intake & 725.1 & 804.7 & 37.87 & 0.18 & 542.6 & 790.1 & 962.0 & 46.38 & $<0.01$ & 0.52 \\
\hline ME intake & 643.7 & 673.8 & 36.28 & 0.57 & 443.1 & 683.1 & 850.1 & 44.43 & $<0.01$ & 0.52 \\
\hline Feces excretion & 283.6 & 416.6 & 27.94 & 0.01 & 327.2 & 369.7 & 353.4 & 34.22 & 0.60 & 0.50 \\
\hline Urine excretion & 18.4 & 61.9 & 1.67 & $<0.01$ & 44.2 & 37.8 & 38.4 & 2.05 & 0.08 & 0.20 \\
\hline Methane emission & 63.0 & 69.0 & 2.68 & 0.15 & 55.2 & 69.2 & 73.5 & 3.28 & $<0.01$ & 0.26 \\
\hline Heat production & 459.3 & 536.7 & 21.12 & 0.03 & 421.5 & 532.1 & 540.4 & 25.87 & 0.01 & 0.15 \\
\hline Energy retention & 184.4 & 137.1 & 36.61 & 0.39 & 21.6 & 151.0 & 309.7 & 44.84 & $<0.01$ & 0.80 \\
\hline \multicolumn{11}{|l|}{ Energy utilization } \\
\hline $\mathrm{DE} / \mathrm{GE}$ & 0.71 & 0.66 & 0.019 & 0.07 & 0.62 & 0.68 & 0.74 & 0.023 & $<0.01$ & 0.91 \\
\hline ME/GE & 0.63 & 0.54 & 0.018 & 0.01 & 0.51 & 0.59 & 0.65 & 0.022 & $<0.01$ & 0.64 \\
\hline ME/DE & 0.88 & 0.83 & 0.008 & $<0.01$ & 0.82 & 0.87 & 0.88 & 0.010 & $<0.01$ & 0.19 \\
\hline
\end{tabular}

and 0.60 for Thai native and Charolais crossbred cattle, respectively. The estimated daily MEm was $388.35 \mathrm{~kJ} / \mathrm{kg} \mathrm{BW}^{0.75}$ and $443.68 \mathrm{~kJ} / \mathrm{kg} \mathrm{BW}{ }^{0.75}$ for Thai native and Charolais crossbred cattle, respectively.

\section{DISCUSSION}

In this study, 350-kg big-bag silo storage of FTMR was found to produce good-quality silage that effectively maintained both nutritive and economic values and remained well preserved for more than four weeks. The FTMR fermentation qualities were characterized by a fermentation profile as follows: low

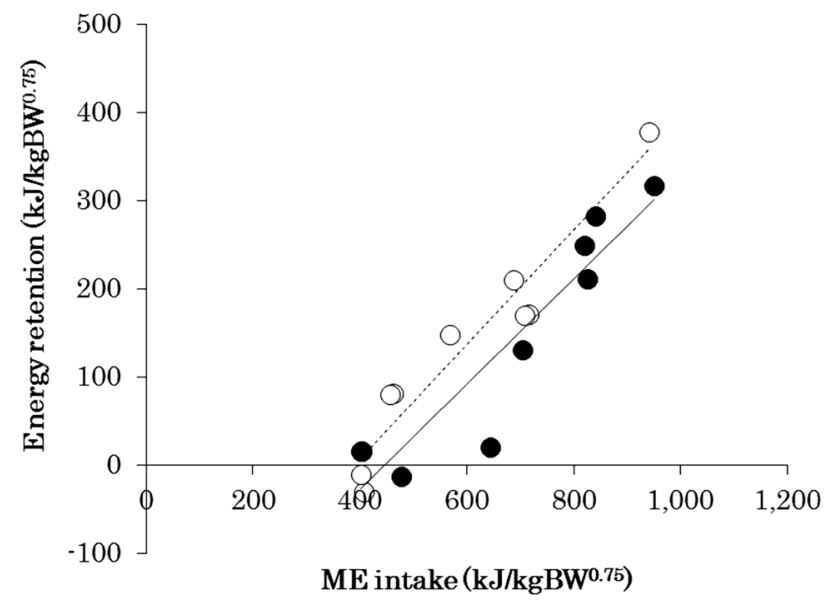

Figure 1. Regression of metabolizable energy (ME) intake on energy retention, scaled for metabolic body weight $\left(\mathrm{kJ} / \mathrm{kg} \mathrm{BW} .15\right.$, where $\mathrm{BW}^{0.75}$ is metabolic body weight). Thai native cattle (o, $n=9$, dashed line): energy retention $=0.65 \times \mathrm{ME}$ intake-252.0 ( $R^{2}=0.92, p<0.001$, residual standard deviation $\left.=36.169\right)$. Charolais crossbred cattle $(\bullet, n=9$, solid line): energy retention $=0.60 \times \mathrm{ME}$ intake-264.4 $\left(R^{2}=0.82, p<0.001\right.$, residual standard deviation $\left.=50.681\right)$.
$\mathrm{pH}, \mathrm{VFA}$ and $\mathrm{NH}_{3}-\mathrm{N}$ values and high lactic acid content (Table 1).

In this study, digestibility possibly differed between breeds because of differences between $B$. indicus and its crossbreeds in terms of anatomy, physiology, and the various microbial populations found in the rumen [5]. This finding was similar to the results of a previous study by Cardenas-Medina et al [18], who demonstrated that there was a higher digestibility of DM in B. indicus than in B. taurus. This may be a result of the higher rate of rumen fermentation in $B$. indicus cattle, due to the different rate at which microorganisms digest cellulose in the rumen. In addition, Ferrell et al [19] make reference to the $B$. indicus breed of cattle utilizing low quality roughage more efficiently than $B$. taurus; these results indicated that the voluntary DM feed intake of beef cattle provided with a tropical feedstuff-based diet is limited to a maximum of $76.3 \mathrm{~g} / \mathrm{kg}$ $\mathrm{BW}^{0.75}$. Feed intake is an important factor to consider because it controls variations in the daily gain of animals [20]. The daily intake of DM and nutrients increases linearly with increasing ME intake levels, resulting in the opportunity for increased growth [2,7].

The present study indicated that feeding level had no effect on nutrient digestibility in cattle. This finding was in contrast to Chaokaur et al [2], who report that the digestibility of DM in Brahman cattle declines significantly with increased feeding level. The ruminal digesta passage rate increases in response to increased feeding levels, reducing the time available for digestion by the rumen microbes. However, the results of the present study were similar to those of Tangitwattanachai et al [7], who report that the digestibility of all nutrients, except for NDF, shows no significant difference when feeding levels are increased in Thai native cattle. Moreover, our results were in 
good agreement with Kaewpila et al [21], who has found that an increased feeding level did not significantly affect the digestibility of $\mathrm{DM}, \mathrm{OM}, \mathrm{CP}, \mathrm{NDF}$, and $\mathrm{ADF}$ in Thai native cattle fed an FTMR diet, indicating that increasing the feeding level in this diet does not alter the digestibility of DM and nutrients in cattle.

In the present study, the enteric methane emission rate (MJ/ $100 \mathrm{MJ}$ GE intake), the methane conversion factor $\left(\mathrm{Y}_{\mathrm{m}}\right)$ were significantly lower in Charolais crossbreeds than in Thai native cattle. $Y_{m}$ value for the two breeds fed an FTMR diet based on good quality tropical feed was near to recommendation by the Intergovernmental Panel on Climate Change [22] (6.5\% \pm $1.0 \%)$. Thai native and Charolais crossbred cattle showed no differences in methane emissions (expressed in $\mathrm{kJ} / \mathrm{kg} \mathrm{BW}^{0.75}$ ). This result was in good agreement with the results of Chuntrakort et al [23], who have determined that methane emissions $\left(\mathrm{kJ} / \mathrm{kg} \mathrm{BW}{ }^{0.75}\right)$ from Thai native cattle were similar to those of Brahman crossbreeds.

Our results indicated that increasing the feeding level drastically reduced the value of the methane conversion factor and enteric methane emission rate (L/kg DM intake, L/kg OM intake, $\mathrm{L} / \mathrm{kg} \mathrm{NDF}$ intake, and L/kg ADG); this result was similar to other reports [2]. These data highlighted the importance of increasing the feeding level to implement an increase in beef cattle performance without change in the cattle population as a strategy in reducing the impacts of global warming, and in improving environmental sustainability in the tropics.

This study demonstrated that an increase in feeding level improves ME intake, ER, and energetic efficiency (Table 4), due to a decrease in the proportion of energy intake to energy excretion in feces, urine, enteric methane, and HP $[2,7,9,10$, 24]. This result indicated a strong relationship in the ME and $\mathrm{DE}$ ratio, which ranged from 0.80 to 0.88 ; the proportion of ME to DE recommended by the ARC [4], National Research Council (NRC) [5], and Commonwealth Scientific and Industrial Research Organisation (CSIRO) [25] is $0.81,0.80$, and 0.82 , respectively. However, the higher range (0.88) exhibited in the $\mathrm{ME}$ to $\mathrm{DE}$ ratio in the present study was within the range of 0.84 to 0.88 suggested by the Chaokaur et al [2], and others $[7,23]$.

The NRC [5] have suggested that energy requirement in $B$. indicus is approximately $10 \%$ less than in B. taurus, while in crossbred cattle (B. indicus $\times$ B. taurus) it is intermediate between the value for the two purebreds. Our study confirmed that MEm in Thai native cattle was lower $14 \%$ less than in Charolais crossbred cattle. Variations in MEm may be affected by physiological conditions of breed, sex, age, physical activity, and the temperature of the environment $[5,6]$. However, the results for MEm in Charolais crossbred cattle $\left(444 \mathrm{~kJ} / \mathrm{kg} \mathrm{BW}^{0.75}\right)$ in the present study were similar to those of Kaewpila et al [21], who reports that MEm in Charolais and Japanese Black crossbred cattle fed in Thailand was $430 \mathrm{~kJ} / \mathrm{kg} \mathrm{BW}^{0.75}$, and 386
$\mathrm{kJ} / \mathrm{kg} \mathrm{BW}{ }^{0.75}$, respectively. CSIRO [25] have also reported that the energy requirement for maintaining $B$. indicus cattle is $20 \%$ lower than for $B$. taurus, because the former have a higher heat tolerance genetic potential for production than the latter. This agrees well with Cardenas-Medina et al [18], who report that, in Mexico, $B$. indicus have a $10 \%$ lower maintenance energy requirement than $B$. taurus; this reduction with increasing body size may be explained by a lower proportion in the weight of the organs and body protein composition.

Efficiency of metabolisable energy utilization requirement for maintenance $\left(\mathrm{k}_{\mathrm{m}}\right)$, the ratio of net energy requirement for maintenance (NEm)/MEm, was used to determine metabolisable energy requirement in beef cattle feeding system worldwide. The results indicated that $\mathrm{k}_{\mathrm{m}}$ for Thai native cattle $\left(\mathrm{k}_{\mathrm{m}}=0.76\right)$ was $4 \%$ higher than for Charolais crossbred cattle $\left(k_{m}=0.73\right)$, which were within the range ( 0.36 to 0.81$)$ for beef cattle recommended by Solis et al [26]. Moreover, WTSR [6] suggested that the $\mathrm{k}_{\mathrm{m}}$ for $B$. indicus (0.64) was higher than the $\mathrm{k}_{\mathrm{m}}$ for $B$. taurus (0.58). The value of $\mathrm{k}_{\mathrm{m}}$ may vary according to variation in NEm which varied and influenced by level of feeding, previous plane of nutrition and breed [5]. Garrett [27] suggested that the variation in $\mathrm{k}_{\mathrm{m}}$ between $B$. indicus and $B$. taurus is affected by body composition and plane of nutrition. The protein turnover may be responsible in variation of $\mathrm{k}_{\mathrm{m}}$ and that the difference of $\mathrm{k}_{\mathrm{m}}$ between $B$. indicus and B. taurus may explain in part of protein turnover. $B$. indicus have less protein turnover than $B$. taurus, which could explain that $B$. indicus have more efficiency in using MEm than B. taurus.

The efficiency of metabolisable energy utilization for growth $\left(\mathrm{k}_{\mathrm{g}}=0.65\right)$ in Thai native cattle was $5 \%$ higher than in Charolais crossbred cattle $\left(\mathrm{k}_{\mathrm{g}}=0.60\right)$; this is similar to the results of Tangjitwattanachai et al [28], who have determined $\mathrm{k}_{\mathrm{g}}$ for $B$. indicus (0.51) to be higher than for B. taurus (0.45). The values of $\mathrm{k}_{\mathrm{g}}$ reported in the present study were very close to the findings of Kaewpila [29] who reports $\mathrm{k}_{\mathrm{g}}$ values of 0.57 and 0.60 for Charolais and Japanese Black crossbred cattle, respectively. The $\mathrm{k}_{\mathrm{g}}$ value provides an estimate of the partial use efficiency of the ratio of the ME for growth to net energy for growth. Energy can be retained in the form of differences in the percentage of energy retention between protein and fat, related to differences in the efficiency of energy utilization. Garrett [27] reports that the efficiency of ME utilization for protein and fat synthesis ranges from $10 \%$ to $40 \%$ and $60 \%$ to $80 \%$, respectively. Thus, these data could indicate that Thai native cattle have a higher degree of physiological maturity, and consequently show better fat deposition, than Charolais crossbred cattle.

The effect of increasing the feeding level on ME utilization for maintenance and growth and the enteric methane conversion factor is shown in Figure 2; increasing the feeding level improved the supply of energy available for growth and reduced the methane conversion factor. This result was in good 


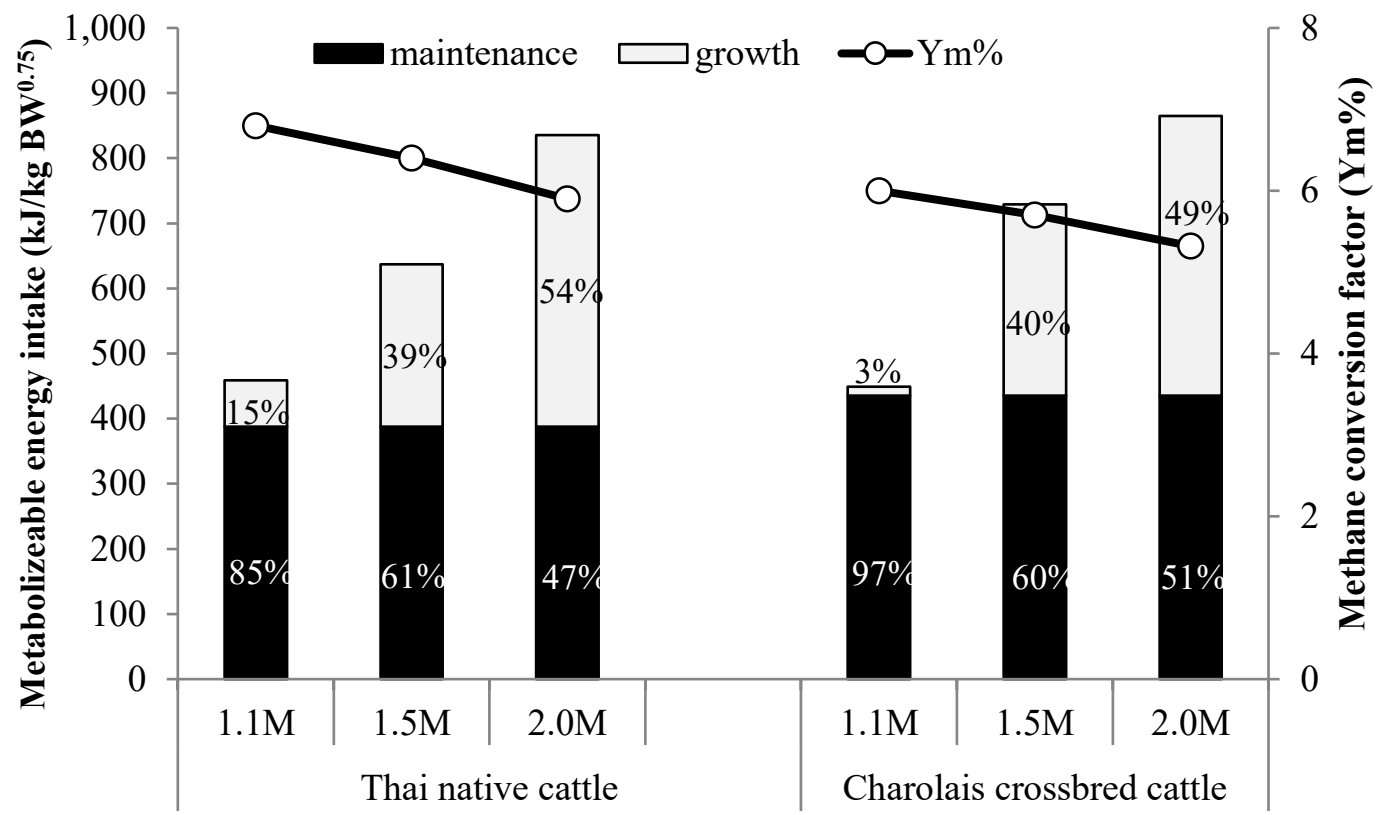

Feeding level

Figure 2. Effect of increasing feeding level (times the metabolizable energy requirement for maintenance [MEm]; $M=388$ and $444 \mathrm{~kJ} / \mathrm{kg} B W^{0.75}$ for Thai native and Charolais crossbred cattle, respectively) on the percentage of metabolisable energy utilization for maintenance and growth to daily metabolizable energy intake (primary horizontal axis, expressed in $\mathrm{kJ} / \mathrm{kg} \mathrm{BW}^{0.75}$ ) and enteric methane emissions energy to gross energy intake or methane conversion factor (Ym) (secondary horizontal axis).

agreement with previous reports [2,7] which have all found that increasing ME intake improves both beef cattle productivity and strategies for enteric methane mitigation $[9,20,23,24]$.

\section{IMPLICATION}

Zebu cattle play an important role in the beef production industry. There is increasing interest in greenhouse gas mitigation strategies to improve the productivity and environmental sustainability of beef production systems. Increasing the feeding level resulted in improved energetic efficiency due to the decreased proportion of energy intake to energy excretion in enteric methane emissions; this therefore improved energy retention in beef cattle fed total mixed ration silage based diet. Methane emissions are not only an important source of greenhouse gases, with major impacts on climate change, but are also a critical factor in the efficiency of feed energy utilization, and are therefore strongly associated with cattle productivity. More feeding trials or on-farm research is needed for the development of a practical and economical zebu crossbred beef farming system.

\section{CONFLICT OF INTEREST}

We certify that there is no conflict of interest with any financial organization regarding the material discussed in the manuscript.

\section{ACKNOWLEDGMENTS}

We thank the Thailand Research Fund, and Khon Kaen University and the Japan International Research Centre for Agricultural Sciences for the infrastructure and laboratory facilities. We also extend thanks to the Khon Kaen University Under Incubation Researcher Project, which supported PhD student, Mr. Sayan Subepang, with a scholarship.

\section{REFERENCES}

1. Gerber PJ, Hristov AN, Henderson B, et al. Technical options for the mitigation of direct methane and nitrous oxide emissions from livestock: a review. Animal 2013;7(Suppl 2):220-34.

2. Chaokaur A, Nishida T, Phaowphaisal I, Sommart K. Effects of feeding level on methane emissions and energy utilization of Brahman cattle in the tropics. Agric Ecosyst Environ 2015; 199:225-30.

3. Ogino A, Sommart K, Subepang S, et al. Environmental impacts of extensive and intensive beef production systems in Thailand evaluated by life cycle assessment. J Clean Prod 2016;112:2231.

4. Agricultural Research Council (ARC). The nutrient requirements of ruminant livestock. Technical Review by an Agricultural Research Council Working Party, Commonwealth Agricultural Bureau, Farnham Royal. UK Press; 1980.

5. National Research Council (NRC). Subcommittee on beef cattle nutrition. Nutrient requirements of beef cattle. 7 th rev. 
ed. Washington, DC, USA: National Academy Press; 2000.

6. The Working Committee of Thai Feeding Standard for Ruminant (WTSR). Nutrient requirement of beef cattle in Indochinese Peninsula. Khon Kaen, Thailand: Klungnanavitthaya Press; 2010.

7. Tangjitwattanachai N, Phaowphaisal I, Otsuka M, Sommart $K$. Enteric methane emission, energetic efficiency and energy requirements for the maintenance of beef cattle in the tropics. Jpn Agric Res Q 2015;49:399-407.

8. Schneider BH, Flatt WP. The evaluation of feeds through digestibility experiments. Athens, GA, USA: University of Georgia Press; 1975.

9. Suzuki T, Phaowphaisal I, Pholsen $\mathrm{P}$, et al. In vivo nutritive value of pangola grass (Digitaria eriantha) hay by a novel indirect calorimeter with a ventilated hood in Thailand. Jpn Agric Res Q 2008;42:123-9.

10. Kongphitee K, Sommart K, Phonbumrung T, Gunha T, Suzuki T. Feed intake, digestibility and energy partitioning in beef cattle fed diets with cassava pulp instead of rice straw. AsianAustralas J Anim Sci 2018;31:1431-41.

11. Cao Y, Takahashi T, Horiguchi K, Yoshida N, Zhou D. In vitro ruminal dry matter digestibility and methane production of fermented total mixed ration containing whole-crop rice and rice bran. Grassl Sci 2012;58:133-9.

12. Fawcett JK, Scott Je. A rapid and precise method for the determination of urea. J Clin Pathol 1960;13:156-9.

13. Porter MG, Murray RS. The volatility of components of grass silage on oven drying and the inter-relationship between drymatter content estimated by different analytical methods. Grass Forage Sci 2001;56:405-11.

14. Association of Official Agricultural Chemists (AOAC). Official methods of analysis of the AOAC International. 16th ed. Washington, MD, USA: AOAC International; 1995.

15. Van Soest PJ, Robertson JB, Lewis BA. Methods for dietary fiber, neutral detergent fiber, and nonstarch polysaccharides in relation to animal nutrition. J Dairy Sci 1991;74:3583-97.

16. Statistical Analysis Systems Institute (SAS). Version 9.1. Cary, NC, USA: SAS Institute Inc.; 2008.

17. Steel RGD, Torrie JH. Principles and Procedures of Statistics. NY USA: McGraw Hill Book Co.; 1980. 633 p.

18. Cardenas-Medina JV, Ku-Vera JC, Magana-Monforte JG. Estimation of metabolizable energy requirements for maintenance and energetic efficiency of weight gain in Bos taurus and Bos indicus cows in tropical Mexico. J Anim Vet Adv 2010;9:5218.

19. Ferrell CL, Jenkins TG, Freetly HC. Feed utilization and performance of crossbred tropically adapted cattle. In: Morrison DG, editor. A compilation of research results involving tropically adapted beef cattle breeds. Baton Rouge, LA, USA: Tropically Adapted Breeds - Regional Project S-1013; 2005. p. 131-47.

20. VandeHaar MJ, Armentano LE, Weigel K, et al. Harnessing the genetics of the modern dairy cow to continue improvements in feed efficiency. J Dairy Sci 2016;99:4941-54.

21. Kaewpila C, Sommart K, Mitsumori M. Dietary fat sources affect feed intake, digestibility, rumen microbial populations, energy partition and methane emissions in different beef cattle genotypes. Animal 2018;12:2529-38.

22. Intergovernmental Panel on Climate Change (IPCC). IPCC Guidelines for National Greenhouse Gas Inventories. Hayama, Japan: Institute for Global Environmental Strategies (IGES); 2006.

23. Chuntrakort P, Otsuka M, Hayashi K, et al. The effect of dietary coconut kernels, whole cottonseeds and sunflower seeds on the intake, digestibility and enteric methane emissions of Zebu beef cattle fed rice straw based diets. Livest Sci 2014;161:80-9.

24. Kongphitee K, Udchachon S, Otsuka M, Sommart K. Energetic efficiency of Thai native beef cattle fed rice straw or Ruzi straw base diet. Khon Kaen Agric J 2010;38(Suppl):176-9.

25. The Commonwealth Scientific and Industrial Research Organisation (CSIRO). Nutrient requirements of domesticated ruminants. Collingwood, Victoria, Australia: CSIRO Publishing; 2007.

26. Solis JC, Byers FM, Schelling GT, Long CR, Greene LW. Maintenance requirements and energetic efficiency of cows of different breed types. J Anim Sci 1988;66:764-73.

27. Garrett WN. Factors influencing energetic efficiency of beef production. J Anim Sci 1980;51:1434-40.

28. Tangjitwattanachai N, Otsuka M, Oshio S, Sommart K. Efficiency of metabolizable energy for maintenance and growth of Bos indicus and Bos taurus beef cattle: a meta-analysis. JIRCAS Work Rep 2009. pp. 40-4.

29. Kaewpila C. Energy utilization and enteric methane emission assessment in beef cattle fed on selected tropical feeds [dissertation]. Khon Kaen, Thailand: Khon Kaen University; 2016. 